\title{
Procesos didácticos en la resolución de PAEV en la institución educativa 82548 Gran Chimú, 2021
}

\author{
Esgardo Vilfredo Lezama León \\ esgaritolezama@gmail.com \\ https://orcid.org/0000-0002-0026-8029 \\ Docente de aula de la I.E. 82548 Jolluco-Gran Chimú \\ Dulio Oseda Gago \\ dosedag@unmsm.edu.pe \\ https://orcid.org/0000-0002-3136-6094 \\ Universidad Nacional Mayor de San Marcos
}

\section{RESUMEN}

El presente estudio se orientó con el objetivo de determinar la influencia de los procesos didácticos en la mejora de la resolución de PAEV en la Institución Educativa 82548 Gran Chimú, 2021.

Por otro lado, el estudio fue de enfoque cuantitativo, según su finalidad, es aplicada y según el alcance o nivel, es explicativo. Presentó un diseño pre experimental con un solo grupo, con pruebas de pre y pos test. La técnica fue utilizada fue la observación y el instrumento prueba escrita. La muestra estuvo constituida por 14 estudiantes de sexto grado.

Los resultados de la prueba de normalidad de Shapiro-Wilk, si $(\alpha<0,05)$, determinaron que la distribución no es normal, por lo tanto, se aplicó las pruebas no paramétricas para muestras relacionadas de Wilcoxon. Los resultados se encuentran al 95\% de confiabilidad, donde arroja el valor de la razón $\mathrm{Z}$ de -3,307 así como el valor de significancia de la prueba es menor de 0,05; en consecuencia, se refuta la hipótesis nula y se admite la hipótesis de investigación. Es decir, se acepta que los procesos didácticos mejoran la resolución de PAEV en la Institución Educativa 82548 Gran Chimú, 2021.

Palabras clave: procesos didácticos, resolución de problemas, competencia, aprendizaje, evaluación. 


\title{
Didactic processes in the resolution of PAEV in the educational institution 82548 Gran Chimú, 2021
}

\begin{abstract}
The present study was oriented with the objective of determining the influence of the didactic processes in the improvement of the resolution of PAEV in the Educational Institution 82548 Gran Chimú, 2021.

On the other hand, the study was of a quantitative approach, according to its purpose, it is applied and according to the scope or level, it is explanatory. It presented a preexperimental design with only one group, with pre and post-test tests. The technique used was observation and the written test instrument. The sample consisted of 14 sixth grade students.

The results of the Shapiro-Wilk normality test, if $(\alpha<0.05)$, determined that the distribution is not normal, therefore, the non-parametric tests for Wilcoxon related samples were applied. The results are at $95 \%$ reliability, where the value of the $\mathrm{Z}$ ratio is -3.307 as well as the significance value of the test is less than 0.05 ; consequently, the null hypothesis is disproved and the research hypothesis is admitted. That is, it is accepted that the didactic processes improve the resolution of PAEV in the Educational Institution 82548 Gran Chimú, 2021.
\end{abstract}

Keywords: didactic processes, problem solving, competence, learning, evaluation.

Artículo recibido: 18. Junio. 2021 Aceptado para publicación: 26. Julio. 2021 Correspondencia: esgaritolezama@ gmail.com Conflictos de Interés: Ninguna que declarar 


\section{INTRODUCCIÓN}

El área curricular de matemática, es un componente primordial de la integralidad formativa de nuestros alumnos, en vista que, forma parte del aprendizaje para la vida. En el desenvolvimiento de nuestra vida diaria hacemos uso de operaciones matemáticas, por ejemplo, cuando queremos adquirir un producto, cuando contabilizamos los años para determinar nuestra edad cronológica, para identificar cuántos somos los miembros de nuestra familia, etcétera; es decir la utilizamos en diferentes circunstancias de nuestra vida.

La problemática, en el ámbito internacional, refleja que, una de las áreas que presentan bajo rendimiento académico es la matemática, específicamente en la resolución de problemas. Al respecto, (PISA, 2019), reporta que los estudiantes evidencian complicaciones cuando se enfrenta a situaciones en la que deba resolver problemas matemáticos; uno de los factores influyentes es la práctica pedagógica, es decir que, persiste la enseñanza tradicional, en donde se gestiona aprendizajes descontextualizados que no responden a la realidad y necesidades de los estudiantes; por otro lado, la secuencia de actividades que se realizan en las sesiones de aprendizaje carecen de procesos didácticos, generando una desarticulación en la secuencia de actividades generadas.

Relacionado a lo anterior, la Organización de las Naciones Unidas para la Educación, la Ciencia y la Cultura, (UNESCO, 2016), señala, los avances generados en los últimos años son insuficientes en vista que, los estudiantes logran predominantemente los niveles de logro más bajos, es decir que no alcanzan los estándares básicos en relación al área. Por otro lado, entre la resolución de problemas y simples y complejos, existe un predominio, aunque con dificultad, para enfrentarse a situaciones de resolución de situaciones simples y deficiente solución de los problemas complejos.

La problemática, en el ámbito nacional, evidencia que, nuestros estudiantes presentan serias dificultades en situaciones en la que tiene que enfrentar la solución de situaciones matemáticas. En esta coyuntura, (MINEDU, 2020), a través de la entidad medición de la calidad, reporta que, nuestros estudiantes presentan un bajo nivel académico en la competencia matemática, reflejado en una deficiente comprensión de las nociones de cantidad, sus operaciones y sus propiedades. Este problema se refleja ante la carente estrategia de enseñanza, es decir, la docencia presenta dificultades en su proceso de planificación cuando establece las estrategias y los recursos a utilizar, generando 
desmotivación y bajo nivel de compromiso por parte de los estudiantes, desencadenando un deficiente rendimiento académico en relación al área curricular.

La problemática, en el ámbito regional, revelan que, nuestros estudiantes obtienen deficientes resultados en las competencias matemáticas. Según la Gerencia Regional de Educación La Libertad (GRELL, 2019), reporta que, en la provincia de Gran Chimú, los informes de la última evaluación censal, reflejan las complicaciones que presentan los estudiantes en la competencia matemática, la misma que, reflejan bajos niveles de logro, lo cual genera ansiedad y frustración en nuestros estudiantes, lo mismo que, desencadena en fracaso escolar.

La problemática, en el ámbito institucional, reflejan que, en la Institución Educativa $\mathrm{N}^{\circ}$ 82548 Dos de mayo, los y las estudiantes reflejan dificultades para la resolución de problemas matemáticos, este diagnóstico responde a la carencia de estrategias y procedimientos de aprendizaje, lo cual afecta su rendimiento académico en el área, así mismo, la carencia de procesos didácticos en la ejecución de las sesiones, profundizan aún más la problemática existente, trayendo como consecuencia, el fracaso escolar, lo cual los desalienta en continuar sus estudios para el logro de su formación integral (Sánchez, 2020).

El presente estudio, en relación a la justificación, se tomará en consideración lo afirmado por, (Hernández y Mendoza, 2018), un estudio se orienta en su relevancia e importancia por diversas causales, una de estas es que, una investigación propone alternativas que permiten resolver una situación cotidiana en el aprendizaje de los estudiantes, otra posibilidad de la relevancia de un estudio, es que aporta al marco teórico, enriqueciendo la literatura científica, otro aspecto que le otorga relevancia a un estudio, es cuando aporta al método científico, es decir, el estudio se convierte en un antecedente para otras investigaciones de similares características; por ejemplo, brinda un instrumento para el recojo de datos que permita observar una variable de estudio, además, brinda un procedimiento nuevo en la metodología científica.

La investigación se enmarca en conveniencia, ante el reflejo del diagnóstico en donde se manifiesta las dificultades presentadas por los estudiantes en la resolución de problemas matemáticos, el presente estudio brindará el conocimiento consistente para establecer las mejoras en el desarrollo de la competencia matemática y de sus procesos didácticos. Por otro lado, el estudio repercute sobre el aprendizaje de los estudiantes, porque la mejoras 
que se propongan, tendrá influencia directa en el rendimiento académico de los educandos (Gómez, 2016).

Por otro lado, el estudio se orienta por sus implicancias prácticas, porque permite conocer el nivel en que se encuentra el aprendizaje de los estudiantes, es decir que ante el diagnóstico que refleja las necesidades y demandas de los educandos, el presente estudio aporta con la información necesaria para brindar el andamiaje a los estudiantes, con el propósito de mejorar su rendimiento, para que adquieran el conocimiento necesario y sean capaces de resolver problemas de su propio contexto social, lo cual genere un efecto que permita desenvolverse adecuadamente en su vida cotidiana (Dieterich, 2016).

Aunado a lo anterior, el presente estudio se orienta en el valor teórico, es decir que la revisión de las principales teorías científicas permitirá realizar una contrastación con los hallazgos obtenidos en el presente estudio, lo cual permitirá enriquecer la literatura científica, la cual podrá ser utilizada por otros estudios con similares características que quieran aplicar alternativas de mejora ante problemáticas existentes en sus entornos educativos (Tacillo, 2017).

Finalmente, el presente estudio, se orienta por su utilidad metodológica, se identifica el estudio sobre el uso de los procesos didácticos como un factor que incide decisivamente en el método científico, es decir, las conclusiones teóricas se constituyen en aporte al método científico porque se utiliza un instrumento que permite solucionar un problema de contexto real, además posterior al estudio se propone alternativas de mejora, así mismo, se propone un marco empírico y teórico que permite consolidar la metodología científica en el presente estudio (Hernández y Mendoza, 2018).

En el presente estudio, para la selección de los antecedentes, se consideró los trabajos con similares características. Así tenemos, en el ámbito internacional, se consideró como ejemplo el estudio realizado por, (Albán, 2018), quien realizó un estudio sobre, una propuesta didáctica que permite a los estudiantes resolver problemas asociados a la matemática en su entorno real y cotidiano. Este estudio tiene como propósito brindar información sobre la influencia que tiene la propuesta diseñada sobre el rendimiento de los estudiantes ligados al campo de la matemática, el estudio es de naturaleza cuantitativa, de un nivel explicativo, cuya muestra está integrada por ciento diez estudiantes, a quienes se les aplicó un instrumento que permitió recoger los siguientes hallazgos, los estudiantes que alcanzaron los más altos niveles de rendimiento fueron quienes recibieron de sus 
maestros mejores herramientas o estrategias metodológicas y los estudiantes que reflejaron deficientes niveles de rendimiento fueron aquellos quienes recibieron de sus docentes escasas herramientas y estrategias didácticas. Este estudio implica que, mientras más herramientas, estrategias y recursos utilicen los docentes, mejores posibilidades de desarrollo y niveles de rendimiento obtendrán de sus estudiantes.

Así mismo, se consideró el estudio realizado por (Fonseca y Jiménez, 2019), quienes realizaron un estudio sobre proponer alternativas didácticas ante situaciones matemáticas que permitan a los estudiantes resolver problemas de su entorno cercano. El estudio se orientó en el propósito de observar que tanto influyen las estrategias en la resolución de situaciones matemáticas. El estudio fue de naturaleza cualitativa, con un alcance descriptivo, la muestra de estudio estuvo integrada por estudiantes de quinto, a quienes se les aplicó un diagnóstico con un instrumento de pre test, para luego aplicar talleres formativos, con los que se pudo observar que en el instrumento de post test mejoraron considerablemente la resolución de situaciones matemáticas de su entorno cotidiano. Este estudio refleja que, si los estudiantes poseen las herramientas y los procedimientos necesarios para enfrentar con éxito situaciones asociadas a las competencias matemáticas, en su entorno, son capaces de demostrar un alto nivel de compromiso y aprendizaje que les permita mejorar considerablemente su rendimiento académico.

En el ámbito nacional, se consideró como antecedente, el trabajo realizado por, (Alvarez, 2019), quien realizó un estudio sobre la utilidad del método Polya en el desarrollo de la competencia matemática de la resolución de problemas de cantidad en estudiantes que presentaban dificultades en el desenvolvimiento de su actuación entrono a situaciones matemáticas. El estudio fue de naturaleza cuantitativa, con un alcance explicativo, la muestra de estudio fueron sesenta estudiantes de quinto grado del nivel primaria, a quienes se les aplicó una prueba de desarrollo para medir las variables de estudio, en un momento previo los estudiantes mostraron un bajo rendimiento en la resolución de situaciones matemáticas, influenciados por la carencia de estrategias y procedimientos matemáticos; en un segundo momento, luego de la aplicación de la estrategia, los estudiantes demostraron tener las herramientas necesarias para enfrentar con éxito las situaciones matemáticas. El estudio refleja que, mientras los docentes otorguen las herramientas y mecanismos necesarios a los estudiantes, estos, actuarán con éxito ante situaciones que les toque enfrentar en su vida cotidiana. 
Aunado a lo anterior, se consideró como antecedente, el estudio realizado por (Javier, 2019), quien realizó un estudio sobre una metodología matemática, el estudio se orientó en el propósito de comprobar el éxito de la propuesta metodológica. El estudio fue de naturaleza cuantitativa, con un alcance explicativo, los sujetos de investigación estuvieron integrados por sesenta estudiantes de Educación primaria, a quienes se les aplicó una evaluación previa para diagnosticar su nivel de aprendizaje para luego desarrollar la propuesta que permita mejorar el diagnóstico existente, posteriormente se aplicó una evaluación posterior la cual determinó claramente el éxito de la propuesta. Este estudio refleja que, las estrategias tienen éxito en los sujetos aprendices porque se aplican respetando sus características, necesidades y demandas, además las situaciones de aprendizajes se extraen del entorno cercano del estudiante haciendo que ellos mismo le encuentren un sentido a su aprendizaje, la cual lo aplicarán en su vida cotidiana.

En el ámbito local, se consideró como antecedente, el trabajo realizado por, (Ruiz, 2020), quien realizó un estudio sobre, programa educativo basado en el Método Polya en el desarrollo de la competencia resolutiva. El estudio se orientó en el propósito de demostrar que tan influyente es la propuesta en los estudiantes que participaron en la investigación. El estudio fue de enfoque y naturaleza cuantitativa con un alcance explicativo. Los sujetos de investigación lo conformaron treinta y cuatro estudiantes de cuarto grado de secundaria, a quienes se le aplicó, como instrumento, una prueba objetiva de resolución de problemas. Los hallazgos determinaron que, el programa Educativo, utilizado como propuesta, fue eficaz y eficiente en el desarrollo de la competencia resolutiva en matemática. Estos resultados demuestran que, la metodología empleada, como estrategia didáctica, es efectiva en el logro de los aprendizajes, además es importante señalar que, las estrategias didácticas deben formar parte de los procesos de planificación, las cuales permitan que la forma de enseñar salga del paradigma tradicional para transitar en una enseñanza que promueva la escuela activa, en donde el estudiante sea protagonista de su experiencia de aprendizaje en el que la significatividad de lo que aprende sea una constante en el proceso de aprendizaje.

Las teorías científicas, en relación a las variables de estudio, le otorgan la consistencia fundamentada, tal es así que, en relación a los procesos didácticos, tenemos a (Polya, 1989), quien sostiene que, son formas estratégicas de gestionar el aprendizaje, generando mayor expectativa de parte de los estudiantes en las diversas formas de aprender. Por otro 
lado, estos procesos deben estar inherentes en los procesos de planificación en el área, los cuales deben responder a las características, necesidades y demandas del sujeto que aprende.

En la misma línea, según, (Cerda, et al., 2017), sostienen que, los procesos didácticos son secuencias de actividades que permiten una mejor gestión del aprendizaje, además es mucho más comprensible y genera mayor expectativa y motivación para el aprendizaje. Además, esta secuencia ordenada y sistemática, permite que las actividades de enseñanza vayan en sintonía con las actividades de aprendizaje.

Los procesos didácticos, según (MINEDU, 2016), los cuales están asociados al desarrollo de las competencias matemáticas y que permiten una mejor gestión de los aprendizajes en el área, se presentan secuencialmente de la siguiente manera:

En primera acción del proceso es que los estudiantes se familiaricen con la situación problemática propuesta, este proceso implica que, el estudiante se apropie del conocimiento a través de la actividad práctica, es decir que, identifica los componentes propios de la situación, los cuales le permitirán tener un panorama de comprensión más simple que le permita tener una mejor acción resolutiva ante la situación presentada. La siguiente acción es la búsqueda e implementación de las herramientas que le permitan enfrentar con éxito la situación, es decir, los estudiantes echarán mano de lo que aprendieron en el proceso y utilizarán las herramientas más eficaces para la resolución de la situación presentada; así mismo una acción que pondrá en juego, en este proceso, es de poner en práctica sus saberes previos, aquellos que lo ayudarán a comprender y resolver exitosamente la situación propuesta. El que los estudiantes aprendan a utilizar las estrategias más pertinentes en la resolución de los problemas matemáticos implica consolidar su aprendizaje autónomo, en el que le permitirá autoevaluarse y retroalimentarse en la mejora de su forma de actuar y aprender.

La tercera acción del proceso es socializar sus representaciones, es decir que, el sujeto que aprende, comparte colaborativamente sus experiencias, las cuales las podrá al servicio del trabajo cooperativo, con el propósito de intercambiar experiencias de aprendizaje con sus pares, en el que la dificultad se resuelva colaborativamente y el éxito del aprendizaje se comparta en la búsqueda de un aprendizaje común. En esta acción es importante que se garantice la presencia de un estudiante, que posea un nivel de aprendizaje satisfactorio 
para que sea el andamiaje de los que necesiten apoyo cognitivo, lo cual repercute en el nivel de aprendizaje de los demás.

El cuarto proceso es la reflexión y formalización, esta acción implica que, los sujetos que aprenden se formulen interrogantes que le permitan comprender la utilidad de lo que aprenden y cómo lo ponen en práctica al servicio de sus contextos. Además, la reflexión contribuye a desafiar al estudiante generando la motivación por aprender y contribuir en la mejora de sus aprendizajes.

Finalmente, el proceso del planteamiento de otros problemas, implica que, el sujeto que aprende tiene la capacidad a partir de la resolución de problemas matemáticos, plantearse nuevas interrogantes que desencadenan nuevas reflexiones y situaciones matemáticas, en las que nuevamente pondrán a prueba los conocimientos adquiridos recuperando sus saberes previos y aplicando los nuevos conocimientos.

Así mismo, (Meneses y Peñaloza, 2019), sostiene que, las situaciones matemáticas deben planificarse considerando las características de los estudiantes y utilizando el contexto en el que se desenvuelven los estudiantes, con el propósito de atender la diversidad del aula, acercando el aprendizaje a sus comunidades y promover el sentido utilitario de lo que aprenden. Un aprendizaje que se gestiona en lo rutinario, es decir que se centra en cuatro paredes, sin la aplicación utilitaria del contexto, es un aprendizaje que se sustenta sin consistencia práctica, por el contrario, si el aprendizaje se aplica en el contexto cercano al estudiante genera un sentido utilitario de lo aprenden.

Las teorías científicas, en relación a la variable resolución de problemas, tenemos a, los Problemas aritméticos elementales verbales (PAEV), al respecto, (MINEDU, 2015), señala que, estas situaciones matemáticas permiten que los estudiantes se apropien de procesos matemáticos, en los que utilizando como escenario su propio contexto socio cultural, permite generar aprendizajes más pertinentes y consistentes. Así mismo, esta propuesta permitirá que los estudiantes se apropien de procesos y propiedades matemáticas, aprendiendo de una manera diferente, puesto que no se privilegia el contenido, sino el sentido utilitario de lo que aprende, para ponerlo al servicio de sus comunidades y de su vida diaria.

Así mismo, (Chamorro, 2003), en la resolución de problemas PAEV señala que, las formas didácticas y el enfoque resolutivo buscan abordar nuevas formas de aprender en 
donde los estudiantes desarrollen competencias y capacidades para solucionar situaciones problemáticas de su vida cotidiana en los diferentes contextos donde convive diariamente. En coherencia a lo anterior, según, (Centeno, 2017), sostiene que, la resolución de problemas, implica la búsqueda de estrategias metodológicas efectivas, que permitan la resolución adecuada de los problemas planteados en la matemática, lo cual desencadena un procedimiento cuyos pasos son sencillos y fáciles de secuenciar.

Así mismo, (Villalobos, 2008), sostiene que, un aprendizaje es consistente y se asocia a la significatividad cuando las estrategias de enseñanza son pertinentes en la planificación, es decir que los propósitos, las estrategias, los recursos, los instrumentos responden a las necesidades y características, así como al contexto sociocultural en el que se desenvuelven los estudiantes. La clave está en la planificación, la cual permite, en relación a lo que se plantea como propósitos, una mejor ejecución de los procesos y generación de aprendizajes más significativos, es por ello que, un docente que planifica de manera pertinente y hace uso de los aspectos más importantes de la planificación como, sus estudiantes, los aprendizaje o propósitos y la didáctica, será un docente que genere aprendizajes apropiados en sus estudiantes.

En razón de lo anterior, (Iriarte, 2011), señala que, las competencias matemáticas, han sido una dificultad incluso para el propio docente, es decir la planificación y su ejecución ha generado dificultades en los docentes del área, es decir que, las estrategias y los recursos no han sido los más apropiados, es por ello que, es importante que la planificación responda a las características y necesidades de los estudiantes y que además las situaciones de aprendizaje se extraigan del contexto del estudiante y que además los materiales y recursos educativos se relaciones con las estrategias y actividades generadoras de aprendizaje; por otro lado, es importante que los instrumentos, respondan a una evaluación auténtica, es decir estén en sintonía con los propósitos y los criterio e indicadores de lo que se desea valorar para retroalimentar el proceso y ayude a mejorar el aprendizaje.

Teniendo en cuenta las consideraciones mostradas anteriormente, el problema general de esta investigación se planteó de la siguiente manera: ¿Cuál es la influencia de los procesos didácticos en la mejora de la resolución de PAEV en estudiantes de sexto grado de la Institución Educativa 82548 Gran Chimú, 2021? Por otro lado, el presente estudio, plantea el siguiente objetivo general: determinar la influencia de los procesos didácticos 
en la mejora de la resolución de PAEV en la Institución Educativa 82548 Gran Chimú, 2021. Finalmente, se formula la siguiente hipótesis general: los procesos didácticos influyen en la mejora de la resolución de PAEV en la Institución Educativa 82548 Gran Chimú, 2021.

\section{MÉTODO}

El presente estudio, según su finalidad, es aplicada porque se ejecuta una propuesta para obtener mejoras en la variable dependiente. Según el alcance o nivel, es explicativo porque brindará las razones de cuál es la influencia que tiene la propuesta sobre la variable problema. Según su diseño es pre experimental, con un solo grupo (Hernández y Mendoza, 2018). El esquema es el siguiente:

\section{GE: $\quad 01 \quad \mathrm{X} \quad \mathrm{O} 2$}

Dónde:

GE: Grupo Experimental

O1: Prueba del pre test (sobre resolución de PAEV)

Manipulación de la variable independiente (Sesiones con procesos didácticos, sobre temáticas del PAEV)

O2: Prueba del post test (sobre resolución de PAEV)

La población muestra, objeto de estudio del presente trabajo, estuvo comprendido por 14 estudiantes de sexto grado de primaria de la Institución Educativa 82548 Gran Chimú, 2021.

\section{RESULTADOS}

A continuación, se presenta los resultados obtenidos en el presente estudio, los cuales se visualizan en las siguientes tablas.

Tabla 1. Niveles de logro de la variable resolución de PAEV en el pre test y pos test del grupo experimental en estudiantes de sexto grado de la Institución Educativa 82548 Gran Chimú, 2021.

GRUPO EXPERIMENTAL

\begin{tabular}{lcccc}
\hline NIVELES DE LOGRO & \multicolumn{1}{c}{ Pre test } & Post test \\
\cline { 2 - 5 } & $\mathbf{n i}$ & $\mathbf{\%}$ & $\mathbf{n i}$ & $\mathbf{\%}$ \\
\hline LOGRADO & 0 & 0.0 & 12 & 85.7 \\
\hline EN PROCESO & 4 & 28.6 & 2 & 14.3 \\
\hline EN INICIO & 10 & 71.4 & 0 & 0.0 \\
\hline TOTAL & 14 & 100.0 & 14 & 100.0
\end{tabular}

Fuente: Base de datos de la prueba escrita para medir niveles de logro de la resolución de PAEV. En la tabla 1, se puede observar que, los resultados en la variable resolución 
de PAEV del grupo experimental, en el pre test son: nivel inicio $71.4 \%$, proceso $28.6 \%$ y logrado $0.0 \%$. Luego de la aplicación de los procesos didácticos, los resultados conseguidos en el post test muestran un progreso considerable, alcanzando un nivel de Logrado en $85.7 \%$, proceso $14.3 \%$ y un nivel de inicio $0.0 \%$.

Hipótesis: Los procesos didácticos influyen en la mejora de la resolución de PAEV en la Institución Educativa 82548 Gran Chimú, 2021.

Nivel de significancia o riesgo: $\alpha=0,05$.

Tabla 2. Prueba de rangos con signo de Wilcoxon para la contrastación de la hipótesis general en la variable resolución de PAEV en el pre test y pos test del grupo experimental en estudiantes de sexto grado de la Institución Educativa 82548 Gran Chimú, 2021.

Estadísticos de prueba ${ }^{a}$

\begin{tabular}{lc} 
& PREEXP - POSEXP \\
\hline$Z$ & $-3,307^{\mathrm{b}}$ \\
\hline Sig. asintótica(bilateral) &, 001 \\
a. Prueba de rangos con signo de Wilcoxon & \\
\hline b. Se basa en rangos negativos. &
\end{tabular}

En la tabla 2, en relación a la contrastación de la hipótesis general. Los resultados se encuentran al 95\% de confiabilidad, donde arroja el valor de la razón Z de -3,307 así como el valor de significancia de la prueba es menor de 0,05 ; en consecuencia, se refuta la hipótesis nula y se admite la hipótesis de investigación. Es decir, se acepta que los procesos didácticos mejoran la resolución de PAEV en la Institución Educativa 82548 Gran Chimú, 2021.

Decisión estadística: Puesto que ( $\mathrm{p}$-valor: 0,001<0,05 y Z=-3,307), en consecuencia, se refuta la hipótesis nula y se admite la hipótesis de investigación.

Conclusión estadística: Se concluye que, los procesos didácticos mejoran la resolución de PAEV en la Institución Educativa 82548 Gran Chimú, 2021.

\section{DISCUSIÓN}

Respecto a la prueba de hipótesis general, puesto que, (p-valor: 0,001<0,05) en consecuencia, se rechaza la hipótesis nula $(\mathrm{Ho})$ y se acepta la hipótesis alterna (Hi), se concluye que, los procesos didácticos mejoran la resolución de PAEV en la Institución Educativa 82548 Gran Chimú, 2021.

Sin duda este antecedente se ajusta precisamente a la influencia de la variable independiente sobre la variable dependiente, ya que según, (Albán, 2018), en su tesis 
doctoral sobre, procesos didácticos para la resolución de problemas matemáticos demuestra que, los procesos didácticos mejoran considerablemente la resolución de problemas matemáticos. Estos resultados revelan que, los estudiantes que alcanzaron los más altos niveles de rendimiento fueron quienes recibieron de sus maestros mejores herramientas o estrategias metodológicas y los estudiantes que reflejaron deficientes niveles de rendimiento fueron aquellos quienes recibieron de sus docentes escasas herramientas y estrategias didácticas. Este estudio implica que, mientras más herramientas, estrategias y recursos utilicen los docentes, mejores posibilidades de desarrollo y niveles de rendimiento obtendrán de sus estudiantes.

Asimismo, los resultados obtenidos también son fortalecidos, en la medida que la teoría de los procesos didácticos de (Polya, 1989), quien sostiene que, son formas estratégicas de gestionar el aprendizaje, generando mayor expectativa de parte de los estudiantes en las diversas formas de aprender. Por otro lado, estos procesos deben estar inherentes en los procesos de planificación en el área, los cuales deben responder a las características, necesidades y demandas del sujeto que aprende.

También tenemos los hallazgos de, (Fonseca y Jiménez, 2019), en su tesis doctoral sobre, estrategias para resolver problemas matemáticos con ideas de Pólya, en grado quinto. Los resultados reflejaron que, las estrategias de resolución de problemas mejoran considerablemente el rendimiento en el área curricular. Este estudio refleja que, si los estudiantes poseen las herramientas y los procedimientos necesarios para enfrentar con éxito situaciones asociadas a las competencias matemáticas, en su entorno, son capaces de demostrar un alto nivel de compromiso y aprendizaje que les permita mejorar considerablemente su rendimiento académico.

Dichos resultados guardan también una correspondencia biunívoca de igual manera con la teoría de (Meneses y Peñaloza, 2019), quienes sostienen que, las situaciones matemáticas deben planificarse considerando las características de los estudiantes y utilizando el contexto en el que se desenvuelven los estudiantes, con el propósito de atender la diversidad del aula, acercando el aprendizaje a sus comunidades y promover el sentido utilitario de lo que aprenden. Un aprendizaje que se gestiona en lo rutinario, es decir que se centra en cuatro paredes, sin la aplicación utilitaria del contexto, es un aprendizaje que se sustenta sin consistencia práctica, por el contrario, si el aprendizaje se aplica en el contexto cercano al estudiante genera un sentido utilitario de lo aprenden. 


\section{CONCLUSIONES}

1) Se ha determinado que, los procesos didácticos mejoran la resolución de PAEV en la Institución Educativa 82548 Gran Chimú, 2021.

2) Se ha determinado que, los procesos didácticos mejoran, la dimensión combinación, de la variable resolución de PAEV en la Institución Educativa 82548 Gran Chimú, 2021 .

3) Se ha determinado que, los procesos didácticos mejoran, la dimensión cambio, de la variable resolución de PAEV en la Institución Educativa 82548 Gran Chimú, 2021.

4) Se ha determinado que, los procesos didácticos mejoran, la dimensión comparación, de la variable resolución de PAEV en la Institución Educativa 82548 Gran Chimú, 2021.

5) Se ha determinado que, los procesos didácticos mejoran, la dimensión igualación, de la variable resolución de PAEV en la Institución Educativa 82548 Gran Chimú, 2021.

\section{REFERENCIAS BIBLIOGRÁFICAS}

Albán, J. (2018). Estrategias que utilizan los estudiantes para la resolución de un problema matemático y su incidencia en el rendimiento académico. [Tesis de posgrado]. Universidad de Cuenca, Cuenca, Ecuador.

Alvarez, M. (2019). Aplicación del método Polya para el desarrollo de la competencia resuelve problemas de cantidad en estudiantes de primaria en la Institución Educativa $\mathrm{N}^{\circ} 156$ Lima - 2019. [Tesis posgrado]. Universidad César Vallejo, Lima.

Centeno, F. (2017). Method of problem solving and academic performance in Mathematical logic. $\quad$ Redalyc, 33(84), 440-470. https://www.redalyc.org/pdf/310/31054991016.pdf

Cerda, G., Pérez, C., Casas, J., y Ortega, R. (2017). Teaching and Learning of Mathematics: The Need for a Multidisciplinary Analysis. Psychology, Society, \& Education, 9(1), 1-10.

Chamorro, M. (2003). Didactics of Mathematics. Madrid, España: Pearson Prentice Hall.

Dieterich, H. (2016). Nueva guía para la investigación Científica. Lima: UCH.

Fonseca, S., y Jiménez, C. (2019). Estrategias para resolver problemas matemáticos con ideas de Pólya, en grado quinto. Educación y Ciencia (22), 427-456.

Gómez, S. (2016). Metodología de la investigación. México: TERCER MILENIO. 
GRELL. (2019). (17 de abril de 2019). Gerencia Regional de Educación La Libertad. Obtenido de Programas presupuestales: http://grell.gob.pe/

Hernández, R., y Mendoza, C. (2018). Metodología de la investigación: Las rutas cuantitativa, cualitativa y mixta. México: MC GRAW HILL.

Iriarte, A. (2011). Development of the competition. Zona Próxima (15), 2-21.

Javier, M. (2019). Método "ABN" en la resolución de problemas de cantidad en estudiantes de primaria, I.E.P Juan Wesley, UGEL 05 - 2019. [Tesis de posgrado]. Universidad César vallejo, Lima.

Meneses, M., y Peñaloza, D. (2019). The Pólya method as a pedagogical strategy to strengthen the competence to solve mathematical problems with basic operations. Zona próxima (31), 8-25.

MINEDU. (2015). ¿Qué y cómo aprenden nuestros estudiantes? Lima: Metrocolor.

MINEDU. (2016) (15 de diciembre de 2016). Ministerio de Educación. Obtenido de Programa

Curricular de Educación secundaria: http://www.minedu.gob.pe/curriculo/pdf/03062016programa-nivel-secundaria-ebr.pdf

MINEDU. (2020) (14 de abril de 2020). Ministerio de Educación. Obtenido de Unidad de Medición de la Calidad: http://umc.minedu.gob.pe/resultadosnacionales2019/\#1582319153363-

13a7d0ea-b260

PISA. (2019). Programa para la Evaluación Internacional de Estudiantes. Madrid: Secretaría General Técnica.

Polya, G. (1989). Cómo plantear y resolver problemas. Trillas.

Ruiz, M. (2020). Programa educativo basado en el Método Polya en las competencias matemáticas en estudiantes de educación secundaria. [Tesis de posgrado]. Universidad Católica de Trujillo, Trujillo.

Sánchez, E. (2020) (15 de agosto de 2020). Resultados de la Evaluación Censal de Estudiantes. (E. Lezama, Entrevistador)

Tacillo, E. (2017). Metodología de la Investigación Científica. Lima: BAUSATE.

UNESCO. (2016). Aportes para la enseñanza de matemática. Santiago de Chile: OREALC. 
Villalobos, X. (2008). Resolución de Problemas Matemáticos: Un Cambio Epistemológico con Resultados Metodológicos. Calidad, Eficacia y Cambio en Educación, 6(3), 36-58. 